\title{
A Pilot Study of Echinocandin Combination with Trimethoprim/Sulfamethoxazole and Clindamycin for the Treatment of AIDS Patients with Pneumocystis Pneumonia
}

\author{
Mengyan Wang $\mathbb{D}^{1,2}$ Guanjing Lang, ${ }^{1}$ Ying Chen, ${ }^{1}$ Caiqin Hu, ${ }^{1}$ Yongzheng Guo, ${ }^{1}$ Ran Tao, ${ }^{1}$ \\ Xiaotian Dong ${ }^{(},{ }^{1}$ and Biao Zhu $\mathbb{( i )}^{1}$ \\ ${ }^{1}$ The Department of Infectious Diseases, State Key Laboratory for Diagnosis and Treatment of Infectious Diseases, \\ Collaborative Innovation Center for Diagnosis and Treatment of Infectious Diseases, National Clinical Research Center for \\ Infectious Diseases, The First Affiliated Hospital, College of Medicine, Zhejiang University, Hangzhou, China \\ ${ }^{2}$ Xixi Hospital of Hangzhou, Hangzhou 310023, China \\ Correspondence should be addressed to Biao Zhu; zhubiao1207@zju.edu.cn
}

Received 28 July 2019; Accepted 15 October 2019; Published 1 December 2019

Academic Editor: Cinzia Milito

Copyright ( 2019 Mengyan Wang et al. This is an open access article distributed under the Creative Commons Attribution License, which permits unrestricted use, distribution, and reproduction in any medium, provided the original work is properly cited.

\begin{abstract}
Background and Objectives. Pneumocystis pneumonia (PCP) is a common opportunistic infection in acquired immune deficiency syndrome (AIDS) patients that continues to result in a high mortality rate. To develop a better treatment strategy and improve PCP prognosis, a cohort study was conducted to evaluate the therapeutic potential of echinocandin treatment for AIDS patients with PCP (AIDS-PCP). Methods. The AIDS-PCP patients were analyzed in our retrospective cohort study that were hospitalized in The First Affiliated Hospital of Zhejiang University during 2013-2018. The antifungal effects of echinocandins were evaluated in two subgroups that were classified by oxygenation as a proxy for the disease state: $\mathrm{PaO}_{2} / \mathrm{FiO}_{2}>200 \mathrm{mmHg}$ and $\mathrm{PaO} / \mathrm{FiO} 2 \leq$ $200 \mathrm{mmHg}$. Intergroup comparisons and survival curves were used to evaluate the effectiveness of the two AIDS-PCP treatment regimens. Results. During the follow-up, 182 AIDS-PCP patients were diagnosed and analyzed in the study. After excluding 55 patients with other superinfections and five patients that were treated with HAART, the remaining 122 patients were enrolled in the study. The group treated with echinocandins combined with trimethoprim-sulfamethoxazole (TMP-SMZ) and clindamycin exhibited a lower mortality rate $(9.62 \%, 5 / 52)$ than did the group with TMP-SMZ and clindamycin treatment $(20 \%, 14 / 70)$. For AIDS-PCP patients in the $\mathrm{PaO}_{2} / \mathrm{FiO}_{2}>200 \mathrm{mmHg}$ subgroup, treatment with echinocandins combined with TMP-SMZ and clindamycin significantly reduced their mortality rate $(4.44 \%(2 / 45)$ vs. $18.18 \%(10 / 55), P=0.035)$. Conclusion. The results of this study indicate that treatment with echinocandins in combination with the standard TMP-SMZ and clindamycin regimen can improve the prognosis and reduce the mortality rate in patients with mild to moderate AIDS-PCP disease.
\end{abstract}

\section{Introduction}

Human pneumocystis pneumonia (PCP) is caused by Pneumocystis jirovecii. In patients with immunodeficiencies or that are undergoing immunosuppression, latent cells will rapidly multiply and destroy alveolar cells, thereby causing interstitial pneumonia [1]. PCP is a life-threatening opportunistic infection [2] that is the most common cause of AIDSrelated deaths $(20.3-47.7 \%$ of all deaths) [3,4]. Although AIDS-related mortality within a year of initiating ART was 7.44 per 100 person-years which has declined owing to the routine use of highly active antiretroviral therapies (HAART), the risk of early death is still as high as $31.8 \%$ [4, 5]. TMP-SMX is the currently used first-line drug for the prevention and treatment of PCP. Alternative drugs include pentamidine, dapsone plus trimethoprim, primaquine plus clindamycin, and atovaquone. In addition, glucocorticosteroid adjuvant therapy can help mechanical ventilation and reduce the mortality rate within patients exhibiting moderate to severe PCP $[6,7]$. Despite advances in the prevention and management of PCP, the serious side effects and drug resistance associated with existing treatment regimens require 
consideration [8]. Increasing numbers of studies have indicated that mutations in dihydropteroate synthase genes may be associated with the emergence of TMP-SMX resistant strains [9]. Alternative candidate drugs including echinocandins like caspofungin have been investigated thoroughly for therapeutic potential in treating PCP. Caspofungin is an antifungal agent that acts on spore cysts by inhibiting the synthesis of $\beta$-(1,3)-D-glucan [10]. Lee et al., Li et al., and $\mathrm{Lu}$ et al. observed that echinocandins in combination with TMP-SMX treatment can improve the prognosis of PCP patients and decrease the associated mortality rate [11-13]. However, there has been little investigation of these effects in AIDS-PCP patients. Consequently, the current study was designed to evaluate the therapeutic potential of a combined echinocandins/TMP-SMX treatment for AIDS-PCP patients.

\section{Materials and Methods}

2.1. Study Population. A total of 182 AIDS-PCP patients were investigated that were hospitalized in The First Affiliated Hospital of Zhejiang University between January 2013 and June 2018. Inclusion criteria include (1) confirmed AIDS diagnosis, (2) age $\geq 18$ years, (3) naive adults with a first episode of AIDS-PCP, and (4) PCP diagnosis. Diagnosis for PCP includes (1) insidious or subacute onset, dry cough, shortness of breath, and increased postactivity and may have fever, progressive dyspnea, and purpura; (2) chest computed tomography suggests increased lung texture, coarse, striplike shadows, or scattered small patchy shadows or diffuse reticular nodular interstitial infiltration or frosted glassy shadows; (3) hypoxemia; (4) elevated level of blood lactate dehydrogenase; and (5) TMP-SMX treatment responding well. Exclusion criteria included: (1) patients that received HAART at the onset of the disease; (2) the presence of other immunocompromised diseases including tumors, congenital diseases, and prior chemotherapy treatment; (3) immune reconstitution inflammatory syndrome (IRIS)patients; (4) the presence of severe allergies or allergies to sulfa drugs; (5) the presence of severe heart, brain, liver, kidney, or other important organ diseases; and (6) the presence of severe blood and endocrine system diseases or past medical history of these diseases. All patients provided informed consent, and this study was approved by the Ethics Committee of The First Affiliated Hospital, College of Medicine, Zhejiang University (reference number 2017471).

To specifically investigate the therapeutic effects of echinocandins in AIDS-PCP patients, 55 patients with other superinfections and five who had begun antiretroviral therapy were excluded. Of the remaining 122 patients, 52 were provided echinocandins/TMP-SMX/clindamycin treatment and 70 patients were treated with TMP-SMX and clindamycin treatment. And all these patients accepted the treatment of glucocorticoid. Patients were divided into two subgroups according to the patient's oxygenation index: $\mathrm{PaO}_{2} / \mathrm{FiO}_{2}>200 \mathrm{mmHg}(n=100)$ and $\mathrm{PaO}_{2} / \mathrm{FiO}_{2} \leq 200 \mathrm{mmHg}(n=22)$. A schematic indicating the work flow of patient inclusion and classification is shown in Figure 1.
2.2. Clinical Information. Clinical data were collected for each subject including demographic characteristics, treatment, superinfections, clinical outcomes, and the results of laboratory tests within $12 \mathrm{~h}$ of admission. The tests comprised blood tests, biochemical assays, D-dimer, ferritin, CRP, LDH, HBDH, CD4 cell counts, and blood gas analyses.

2.3. Statistical Methods. Nonparametric Mann-Whitney $U$ tests or Student's $t$-tests were used for data analysis when data were nonnormal or normal, respectively. KolmogorovSmirnov tests were used for normal distribution. Chisquared tests, continuity corrections, or Fisher's exact tests were used to test statistical significances for categorical data. Binary logistic regressions were used in multivariate analyses to predict mortality. Kaplan-Meier and log-rank tests were used to analyze and compare survival rates. All statistical analyses were performed using SPSS version 19 (SPSS, Armonk, New York, USA) using a statistical significance threshold of $P<0.05$.

\section{Results}

3.1. Demographic Characteristics and Mortality. A total of 182 AIDS-PCP patients were admitted to The First Affiliated Hospital of Zhejiang University between January 2013 and June 2018. Of the remaining 122 patients, 52 were provided echinocandins/TMP-SMX/clindamycin treatment and 70 patients were treated with TMP-SMX and clindamycin treatment. Clinical characteristics and baseline demographics of the 122 patients between combined echinocandins and noncombined echinocandins are shown in Table 1.

3.2. Intergroup Comparisons of Echinocandins/TMPSMX/Clindamycin and TMP-SMX/Clindamycin Treatments. Among all 122 patients investigated in the study, those within the echinocandins/TMP-SMX and clindamycin treatment group exhibited a mortality rate of $9.62 \%$ (5/52), while those within the TMP-SMX and clindamycin treatment group exhibited a mortality rate of $20 \%$ (14/70). Despite the difference in mortality outcomes, the difference was not statistically significant $(P=0.118$; Figure 2$)$.

In the subgroup of patients with $\mathrm{PaO}_{2} / \mathrm{FiO}_{2} \leq 200 \mathrm{mmHg}$ $(n=22)$, those with echinocandins/TMP-SMX and clindamycin treatment exhibited a mortality rate of $26.67 \%$ (4/15), while the rate was higher, $42.86 \%$ (3/7), for those within the TMP-SMX and clindamycin treatment group. However, the difference between the two treatment groups was not statistically significant $(P=0.630)$.

In the subgroup of patients with $\mathrm{PaO}_{2} / \mathrm{FiO}_{2}>200 \mathrm{mmHg}$ $(n=100)$, those within the echinocandins/TMP-SMX and clindamycin treatment group exhibited a significantly lower mortality rate $(4.44 \%, 2 / 45)$ than did those in the TMPSMX and clindamycin group $(18.18 \%, 10 / 55 ; P=0.035)$.

3.3. Survival in Patients with Mild AIDS-PCP. Survival rates were analyzed and compared between patients in the echinocandins/TMP-SMX/clindamycin and TMP-SMX/clindamycin treatment groups based on clinical outcomes within two months for patients with $\mathrm{PaO}_{2} / \mathrm{FiO}_{2}>200 \mathrm{mmHg}$. Patients within the TMP-SMX/clindamycin treatment group exhibited 


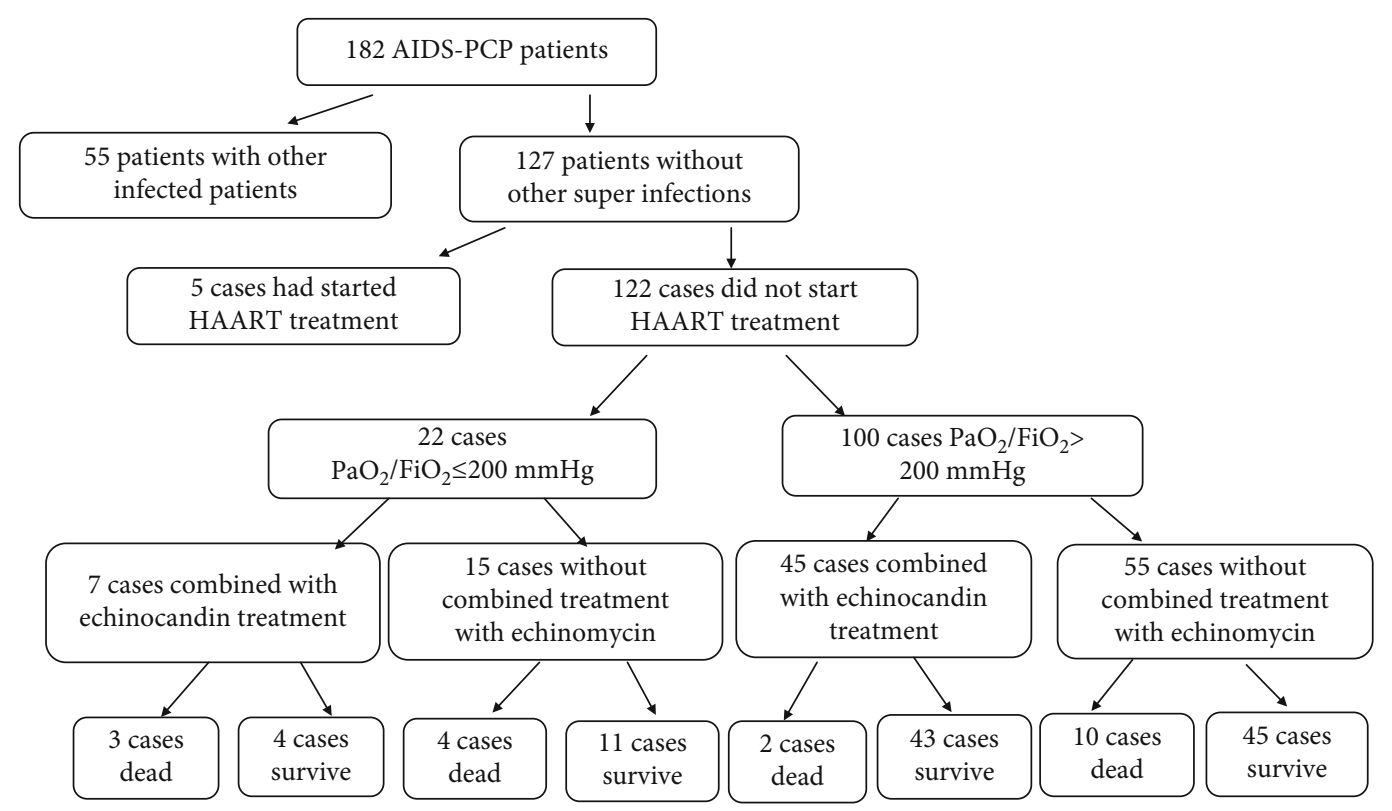

FIGURE 1: Flow chart indicating the process underlying patient selection and group classification.

TABLE 1: Clinical and demographic characteristics of AIDS-PCP patients investigated in this study, China, 2013-2018.

\begin{tabular}{|c|c|c|c|c|}
\hline Demographic data & $\begin{array}{l}\text { Total patients } \\
\quad(n=122)\end{array}$ & $\begin{array}{c}\text { Patients, no. } \\
\text { Combined } \\
\text { echinocandins }(n=52)\end{array}$ & $\begin{array}{c}\text { Noncombined } \\
\text { echinocandins }(n=70)\end{array}$ & $P$ value \\
\hline $\operatorname{Sex}(M / F)$ & $112 / 10$ & $45 / 7$ & $67 / 3$ & 0.096 \\
\hline Age (y) & $42.15 \pm 13.90$ & $41.43 \pm 12.99$ & $43.31 \pm 15.29$ & 0.462 \\
\hline Recent CD4 count (cells/ $\mu \mathrm{L})$ & $59.24 \pm 66.86$ & $64.64 \pm 66.43$ & $51.24 \pm 67.35$ & 0.275 \\
\hline Number of hospitalization days & $19.93 \pm 9.73$ & $19.36 \pm 10.42$ & $20.92 \pm 8.45$ & 0.409 \\
\hline \multicolumn{5}{|l|}{ Treatment } \\
\hline TMP-SMX (80 mg-400 mg, tid) & 122 & 52 & 70 & NA \\
\hline Clindamycin (800 mg, q8h) & 122 & 52 & 70 & NA \\
\hline Echinocandins (50 mg, qd) & 52 & 52 & 0 & NA \\
\hline Glucocorticoid (40 mg, bid) & 122 & 52 & 70 & NA \\
\hline \multicolumn{5}{|l|}{ Clinical outcome } \\
\hline $\mathrm{PaO}_{2} / \mathrm{FiO}_{2}>200 \mathrm{mmHg} / \mathrm{PaO}_{2} / \mathrm{FiO}_{2} \leq 200 \mathrm{mmHg}$ & $100 / 22$ & $45 / 7$ & $55 / 15$ & 0.258 \\
\hline Survival/death & $103 / 19$ & $47 / 5$ & $56 / 14$ & 0.118 \\
\hline
\end{tabular}

a significantly lower survival rate than did those in the echinocandins/TMP-SMX/clindamycin group $(P=0.0384$; Figure 3).

\section{Discussion}

Most AIDS-PCP patients are in advanced stages and have high risks of contracting various opportunistic infections [14]. To adequately investigate the role of echinocandins and TMP-SMX treatments and reduce confounding factors caused by other superinfections and the corresponding treatments, we excluded patients with superinfections from the study cohort. These infections included tuberculosis, Cryptococcus, CMV, Epstein-Barr virus, hepatitis B virus, and syphilis. The glucocorticoid can improve clinical outcome in AIDS patients with PCP. All patients investigated in this study accepted treatment of glucocorticoid for reducing factors of the impacting clinical outcome.

Echinocandins inhibit the synthesis of $\beta$ - $(1,3)$-D-glucan and block the formation of pneumocystis cysts, although they are less effective against trophozoite forms [15]. These observations suggest that echinocandins can reduce pathogen reservoirs. In addition, TMP-SMX inhibits trophozoite forms of Pneumocystis by interfering with their metabolism of folate. Therefore, the combination of echinocandins and TMP-SMX can inhibit the entire life cycle of Pneumocystis parasites [16]. TMP-SMX acts slowly, requiring five to eight days to achieve stable therapeutic effects [17], while echinocandins act rapidly. Consequently, the combination of TMP-SMX and echinocandins can exhibit synergistic effects in the treatment of PCP patients. Importantly, echinocandins induce less adverse events in PCP patients 


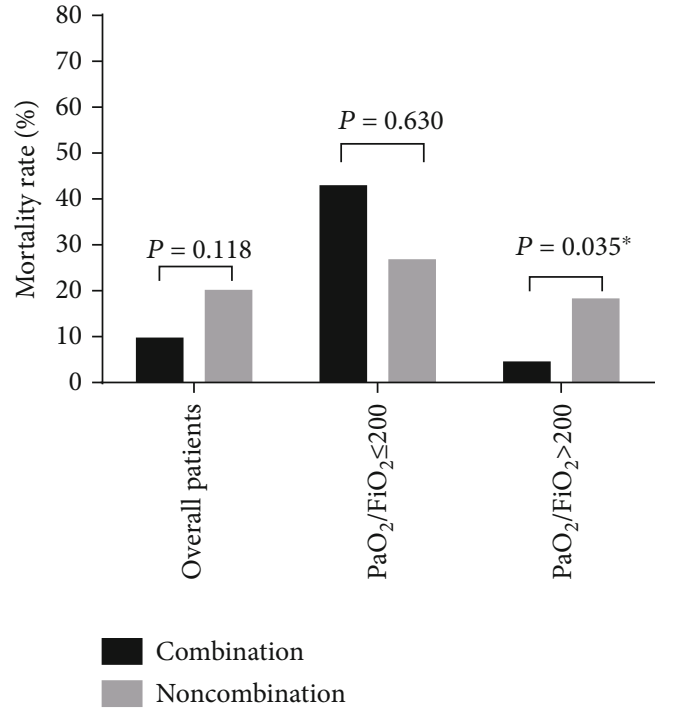

Figure 2: Mortality rates of patients with treatments of either combined echinocandins or noncombined echinocandins.

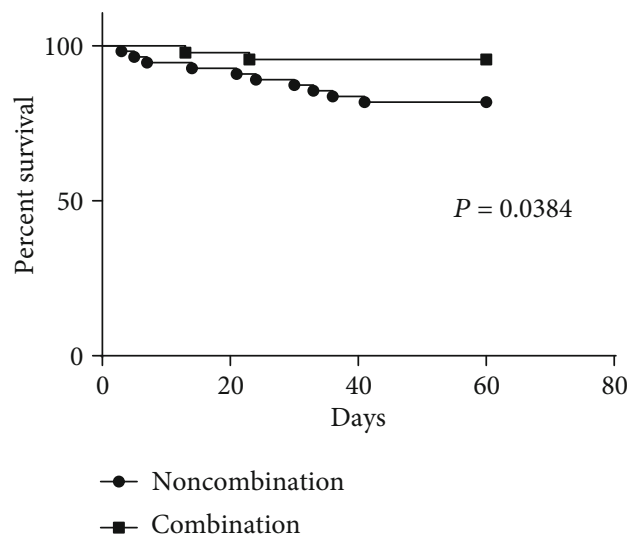

FIgURE 3: Survival in AIDS-PCP patients exhibiting mild disease states $\left(\mathrm{PaO}_{2} / \mathrm{FiO}_{2}>200 \mathrm{mmHg}\right)$ after treatment. Two subgroups were considered based on either combined echinocandin treatment or noncombined echinocandins.

because they do not inhibit the CYP system or induce CYP3A4 drug metabolism [18].

The addition of echinocandins to a standard TMP-SMX regimen reduced the mortality rate among all 122 enrolled patients of this study ( $9.62 \%$ vs. $20 \%$ ), although the difference was not statistically significant. Following this observation, patients were divided into two subgroups, $\mathrm{PaO}_{2} / \mathrm{FiO}_{2}>200$ $\mathrm{mmHg}$ (indicating mild disease) and $\mathrm{PaO}_{2} / \mathrm{FiO}_{2} \leq 200$ $\mathrm{mmHg}$ (indicating severe disease). The early application of a combined echinocandins/TMP-SMX treatment for mild AIDS-PCP patients could significantly reduce the mortality rate compared to TMP-SMX treatment alone $(4.44 \%$ vs. $18.18 \%, P<0.05)$. These findings are consistent with some case reports suggesting that echinocandins could improve the prognosis of patients with PCP [11-13]. In addition, the feasibility of combined echinocandins/TMP-SMX treatment for PCP was demonstrated in studies of PCP animal models and in vitro experiments [19-21]. A previous caspofungin salvage treatment trial indicated that caspofungin could improve the prognosis and reduce the mortality rate of AIDS-PCP patients [22]. However, there were two major limitations of this study: the lack of a control group and the small sample size $(n=12)$. However, the results of the present study confirm the observations from the trial, while using a larger sample size $(n=122)$ and an experimental design that featured a control group.

In our study, the subgroup of patients with $\mathrm{PaO} 2 / \mathrm{FiO} 2$ $\leq 200 \mathrm{mmHg}(n=22)$ and those with echinocandins/TMPSMX and clindamycin treatment exhibited a mortality rate of $42.86 \%$ (3/7), while the rate was higher, $26.67 \%(4 / 15)$, for those within the TMP-SMX and clindamycin treatment group. This results reported here indicate that adding echinocandins to the standard regimen could not reduce the mortality rate for critically ill patients in advanced stages. However, the sample size of patients with $\mathrm{PaO} 2 / \mathrm{FiO} 2 \leq 200$ $\mathrm{mmHg}$ was small. Lung damage resulting from PCP is very rapid, and deaths often result from a rapid increase in proinflammatory cytokines [23]. Echinocandins can inhibit the inflammatory response induced by $\beta$-glucan by inhibiting $\beta$-glucan synthesis, thereby alleviating the symptoms of PCP [24]. Consequently, early application of a combined echinocandins/TMP-SMX treatment for PCP patients with mild to moderate disease states could inhibit the inflammatory response and cytokine production, thereby reducing their mortality rate.

Our study had some limitations: this study did not perform bronchoscopic examination to make a molecular test for PCP diagnosis. However, most patients were severe. Because of hypoxia and dyspnea symptoms, it was difficult to carry out bronchoscopic examination. And we had adopted a unified clinical diagnostic standard. Additionally, limited sample size, single-center analysis, and retrospective studies may cause some bias.

\section{Conclusions}

The results of this study demonstrate that early application of combined echinocandins/TMP-SMX treatment for AIDSPCP patients can improve patient prognosis, increase their survival rate, and decrease their mortality rate.

\section{Data Availability}

The data statement support the findings of this study are available.

\section{Conflicts of Interest}

The authors declare that they have no competing interests.

\section{Acknowledgments}

We would like to thank the native English-speaking scientists of LetPub Company for editing our manuscript. This work was supported by the National Special Research Program for Important Infectious Diseases (grant numbers 2017ZX10202102-002-002 and 2018ZX10715-014-004). 


\section{References}

[1] C. F. Thomas Jr. and A. H. Limper, "Pneumocystis pneumonia," The New England Journal of Medicine, vol. 350, no. 24, pp. 2487-2498, 2004.

[2] R. O. Oladele, A. A. Otu, M. D. Richardson, and D. W. Denning, "Diagnosis and management of pneumocystis pneumonia in resource-poor settings," Journal of Health Care for the Poor and Underserved, vol. 29, no. 1, pp. 107-158, 2018.

[3] R. N. Wickramasekaran, M. P. Jewell, F. Sorvillo, and T. Kuo, "The changing trends and profile of pneumocystosis mortality in the United States, 1999-2014," Mycoses, vol. 60, no. 9, pp. 607-615, 2017.

[4] S. H. Lee, K. H. Kim, S. G. Lee et al., "Trends of mortality and cause of death among HIV-infected patients in Korea, 19902011," Journal of Korean Medical Science, vol. 28, no. 1, pp. 67-73, 2013.

[5] R. A. Silverman, G. C. John-Stewart, I. A. Beck et al., "Predictors of mortality within the first year of initiating antiretroviral therapy in urban and rural Kenya: a prospective cohort study," PLoS One, vol. 14, no. 10, 2019.

[6] J. G. Castro and M. Morrison-Bryant, "Management of Pneumocystis Jirovecii pneumonia in HIV infected patients: current options, challenges and future directions," HIVIAIDS Research and Palliative Care, vol. 2, pp. 123-134, 2010.

[7] S. A. Bozzette, F. R. Sattler, J. Chiu et al., "A controlled trial of early adjunctive treatment with corticosteroids for Pneumocystis carinii pneumonia in the acquired immunodeficiency syndrome. California Collaborative Treatment Group," The New England Journal of Medicine, vol. 323, no. 21, pp. 14511457, 1990.

[8] Y. S. Huang, J. J. Yang, N. Y. Lee et al., "Treatment of Pneumocystis jirovecii pneumonia in HIV-infected patients: a review," Expert Review of Anti-Infective Therapy, vol. 15, no. 9, pp. 873892, 2017.

[9] O. Matos and F. Esteves, "Epidemiology and clinical relevance of Pneumocystis jirovecii Frenkel, 1976 dihydropteroate synthase gene mutations," Parasite, vol. 17, no. 3, pp. 219-232, 2010.

[10] F. Esteves, F. J. Medrano, Y. de Armas, G. Wissmann, E. J. Calderon, and O. Matos, "Pneumocystis and pneumocystosis: first meeting of experts from Latin-American and Portuguese-speaking countries - a mini-review," Expert Review of Anti-Infective Therapy, vol. 12, no. 5, pp. 545548, 2014.

[11] Y. M. Lu, Y. T. Lee, H. C. Chang et al., "Combination of echinocandins and trimethoprim/sulfamethoxazole for the treatment of Pneumocystis jiroveci pneumonia after heart transplantation," Transplantation Proceedings, vol. 49, no. 8, pp. 1893-1898, 2017.

[12] H. Li, H. Huang, and H. He, "Successful treatment of severe Pneumocystis pneumonia in an immunosuppressed patient using caspofungin combined with clindamycin: a case report and literature review," BMC Pulmonary Medicine, vol. 16, no. 1, p. 144, 2016.

[13] N. Lee, D. Lawrence, B. Patel, and S. Ledot, "HIV-relatedPneumocystis jiroveciipneumonia managed with caspofungin and veno-venous extracorporeal membrane oxygenation rescue therapy," BML Case Reports, vol. 2017, 2017.

[14] K. Buchacz, B. Lau, Y. Jing et al., "Incidence of AIDS-defining opportunistic infections in a multicohort analysis of HIV- infected persons in the United States and Canada, 20002010," The Journal of Infectious Diseases, vol. 214, no. 6, pp. 862-872, 2016.

[15] R. Vassallo, J. E. Standing, and A. H. Limper, "IsolatedPneumocystis cariniiCell wall glucan provokes lower respiratory tract inflammatory responses," Journal of Immunology, vol. 164, no. 7, pp. 3755-3763, 2000.

[16] R. Utili, E. Durante-Mangoni, C. Basilico, A. Mattei, E. Ragone, and P. Grossi, "Efficacy of caspofungin addition to trimethoprim-sulfamethoxazole treatment for severe pneumocystis pneumonia in solid organ transplant recipients," Transplantation, vol. 84, no. 6, pp. 685-688, 2007.

[17] A. Roux, F. Gonzalez, M. Roux et al., "L'infection pulmonaire a _Pneumocystis jirovecii_chez les patients VIH n egatifs : mise au point," Médecine et Maladies Infectieuses, vol. 44, no. 5, pp. 185-198, 2014.

[18] L. Waters and M. Nelson, "The use of caspofungin in HIVinfected individuals," Expert Opinion on Investigational Drugs, vol. 16, no. 6, pp. 899-908, 2007.

[19] M. A. Powles, P. Liberator, J. Anderson et al., "Efficacy of MK991 (L-743,872), a semisynthetic pneumocandin, in murine models of Pneumocystis carinii," Antimicrobial Agents and Chemotherapy, vol. 42, no. 8, pp. 1985-1989, 1998.

[20] M. T. Cushion and M. S. Collins, "Susceptibility of Pneumocystis to echinocandins in suspension and biofilm cultures," Antimicrobial Agents and Chemotherapy, vol. 55, no. 10, pp. 4513-4518, 2011.

[21] M. L. Lobo, F. Esteves, B. de Sousa et al., "Therapeutic potential of caspofungin combined with trimethoprimsulfamethoxazole for pneumocystis pneumonia: a pilot study in mice," PLoS One, vol. 8, no. 8, 2013.

[22] D. Armstrong-James, J. Stebbing, L. John et al., "A trial of caspofungin salvage treatment in PCP pneumonia," Thorax, vol. 66 , no. 6 , pp. 537-538, 2011.

[23] H. Kobayashi, S. Worgall, T. P. O'Connor, and R. G. Crystal, "Interaction of Pneumocystis carinii with dendritic cells and resulting host responses to P. carinii," Journal of Immunother$a p y$, vol. 30, no. 1, pp. 54-63, 2007.

[24] M. J. Linke, A. Ashbaugh, M. S. Collins, K. Lynch, and M. T. Cushion, "Characterization of a distinct host response profile to Pneumocystis murina asci during clearance of pneumocystis pneumonia," Infection and Immunity, vol. 81, no. 3, pp. 984-995, 2013. 


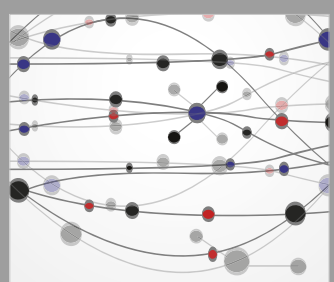

The Scientific World Journal
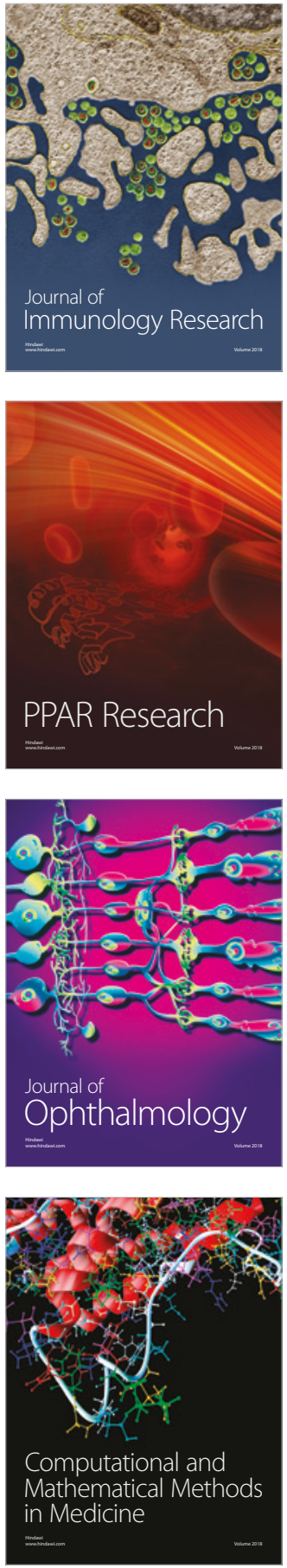

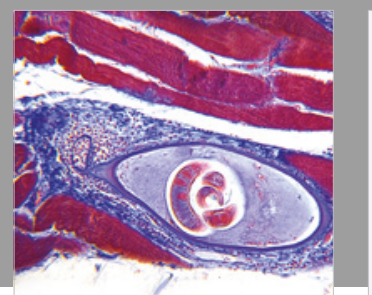

Gastroenterology Research and Practice

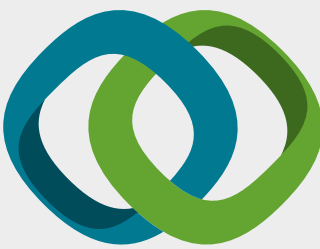

\section{Hindawi}

Submit your manuscripts at

www.hindawi.com
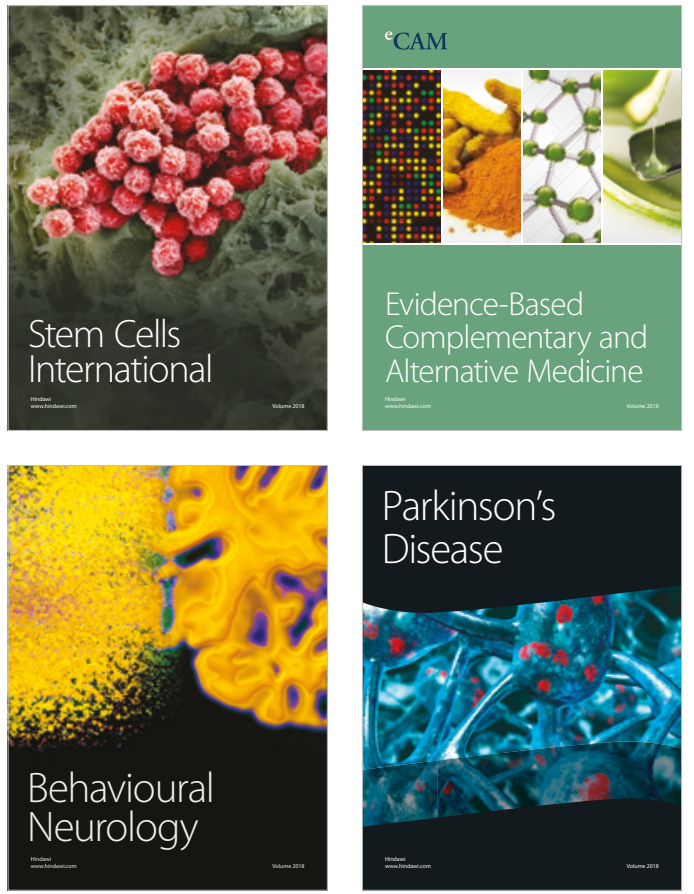

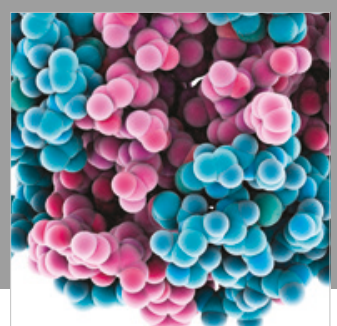

ournal of

Diabetes Research

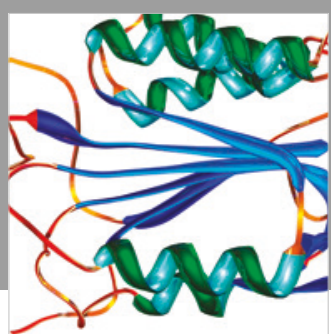

Disease Markers
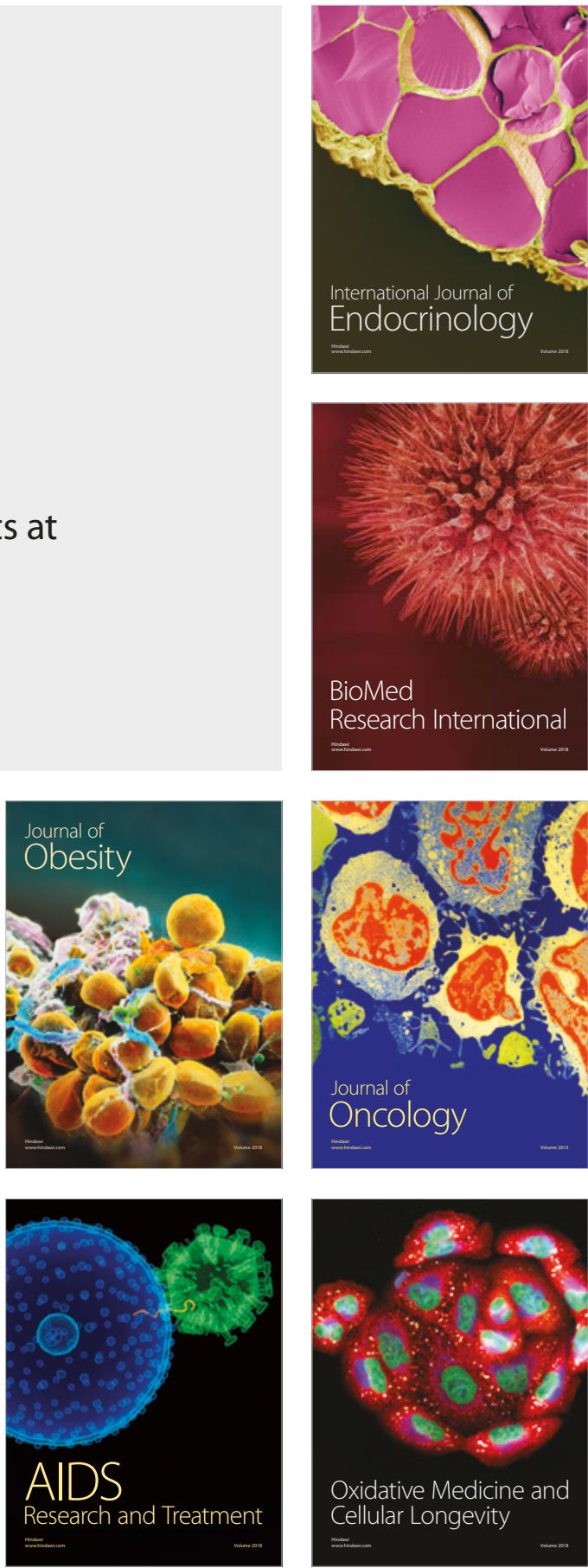